BMJ Open

Diabetes

Research

\& Care

\title{
Positive reinforcement by general practitioners is associated with greater physical activity in adults with type 2 diabetes
}

\author{
Ralph Geerling, ${ }^{1,2}$ Jessica L Browne, ${ }^{2,3}$ Elizabeth Holmes-Truscott, ${ }^{1,2}$ John Furler, ${ }^{4}$
} Jane Speight (1) , ${ }^{1,2}$ Kylie Mosely ${ }^{5,6}$

\begin{abstract}
To cite: Geerling R, Browne JL, Holmes-Truscott E, et al. Positive reinforcement by general practitioners is associated with greater physical activity in adults with type 2 diabetes. BMJ Open Diab Res Care 2019;7:e000701. doi:10.1136/ bmjdrc-2019-000701
\end{abstract}

Received 24 May 2019 Revised 25 September 2019 Accepted 7 0ctober 2019

Check for updates

(c) Author(s) (or their employer(s)) 2019. Re-use permitted under CC BY-NC. No commercial re-use. See rights and permissions. Published by BMJ.

${ }^{1}$ School of Psychology, Deakin University, Geelong, Victoria, Australia

${ }^{2}$ The Australian Centre for Behavioural Research in Diabetes, Melbourne, Victoria, Australia

${ }^{3}$ Centre for Evidence and Implementation, Melbourne, Victoria, Australia

${ }^{4}$ Department of General Practice, The University of Melbourne, Melbourne, Victoria, Australia

${ }^{5}$ BodyMatters Australasia, Sydney, New South Wales, Australia

${ }^{6}$ University of Technology Sydney, Sydney, New South Wales, Australia

Correspondence to Mr Ralph Geerling; rgeerling@acbrd.org.au

\section{ABSTRACT}

Objective In a sample of adults with type 2 diabetes mellitus (T2DM), the aim of this study was to examine whether self-reported physical activity level is associated with recall of specific physical activity-related interactions used by general practitioners (GP).

Research design and methods Adults with T2DM completed an online survey reporting physical activity behaviors and recall of $14 \mathrm{GP}$-patient interactions about physical activity, mapped onto discrete behavior change techniques (BCT). Stepped logistical regression examined associations between recommended physical activity ( $\geq 600 \mathrm{MET}$-min/week) and GP-patient interactions, controlling for body mass index, diabetes-related comorbidities, depressive symptoms and self-efficacy. Results In total, 381 respondents (55\% men, mean \pm SD age: $62 \pm 10$ years and T2DM duration $8 \pm 8$ years) provided complete data. Most (73\%) reported receiving 'general advice', while interactions related to goal setting, monitoring, and relapse prevention were least commonly reported (all $<20 \%$ ). Self-reported achievement of the recommended physical activity level was significantly associated with recall of GP interactions involving praise for 'efforts to be active' (OR 2.1; 95\% $\mathrm{Cl} 1.24$ to 3.53 ), 'Iost weight' (OR 1.81; $95 \% \mathrm{Cl} 1.05$ to 3.12) or lowering 'glucose levels as a result of being active' (OR 1.75; 95\% Cl 1.03 to 2.96).

Conclusions Findings suggest GPs can be somewhat effective in promoting physical activity with simple, positive, reinforcing messages/interactions. Future research to develop and evaluate very brief primary care BCT-based physical activity interventions is needed.

\section{INTRODUCTION}

In people with type 2 diabetes mellitus (T2DM), physical activity has been shown conclusively to improve blood glucose levels, ${ }^{12}$ maintain a healthy weight, ${ }^{3}$ reduce cardiovascular risks ${ }^{4}$ and reduce overall mortality, ${ }^{5}$ as well as reducing depressive symptoms and improving quality of life. ${ }^{6}$ National and international T2DM management guidelines advocate for a minimum of $150 \mathrm{~min}$ of moderate-intensity aerobic physical activity spread across most, if not all, days

\section{Significance of this study}

What is already known about this subject?

- Only one-third of adults with type 2 diabetes mellitus meet physical activity recommendations.

- General practitioners (GPs) consider physical activity counseling important but report several challenges to providing it.

What are the new findings?

- Significantly more adults with type 2 diabetes who met the guidelines for physical activity recalled receiving praise and encouragement from their GP.

- Praising the behavior rather than the outcome may be a more beneficial technique.

How might these results change the focus of research or clinical practice?

- GPs may be able to promote greater physical activity through simple, brief and positive reinforcement.

of the week, ${ }^{78}$ which is equivalent to 600 METmin. However, it is estimated that only onethird of adults with T2DM meet these physical activity recommendations and that inactivity is responsible for $7 \%$ of the burden of disease caused by all types of diabetes globally. ${ }^{5}$

The complex, chronic and progressive nature of T2DM requires ongoing multidisciplinary clinical support, including regular diabetes consultations with health professionals (recommended every 3-12 months). ${ }^{7}$ The majority of T2DM clinical care is provided in the primary care setting, ${ }^{7}$ and the role of the general practitioner (GP) includes monitoring of clinical outcomes, complication risk, emotional well-being, timely review and prescription of treatment, and facilitation of diabetes self-management education and support. From diagnosis, irrespective of treatment progression, GPs should provide ongoing education, goal setting and 
monitoring of physical activity, including discussion of risks, benefits and safety advice. ${ }^{7}$

There is evidence that people with T2DM attending appointments with GPs who use counseling principles within their consultations (eg, person centered, compassionate, respectful, unrushed) are more likely to achieve the recommended level of physical activity. ${ }^{9-11}$ Research suggests that the majority of GPs perceive physical activity counseling to be important and within the scope of their role. ${ }^{12}$ However, GPs report several challenges to providing physical activity counseling, including systemic barriers (eg, lack of time, resources or counseling protocols/guidelines) and professional barriers (eg, lack of knowledge and skills). ${ }^{12}$ Lifestyle counseling may not be prioritized when trying to address the various and complex aspects of managing diabetes, as well as other comorbidities, and other concerns/priorities of the person with T2DM within a single appointment. ${ }^{13}$ GPs may perceive that they are left with little time to discuss the need for lifestyle modifications such as physical activity, or to help motivate patients to be more active. ${ }^{13}$ Evidence suggests that GPs may also feel uncomfortable providing detailed or authoritative advice about physical activity (eg, 'exercise prescriptions'). ${ }^{12}$

By avoiding, delaying or minimizing discussion of physical activity within primary care, there are missed opportunities to raise awareness of its benefits, identify barriers to reaching recommended targets, and support people with T2DM to increase their physical activity level. Previous research has demonstrated the effectiveness of primary care-based behavioral interventions, compared with usual care, in increasing physical activity among people with T2DM. ${ }^{9}$ A study incorporating the findings of a systematic review of 17 interventions identified the behavior change techniques (BCT) that were associated with increased physical activity. ${ }^{9}$ BCTs are 'observable and replicable components of behaviour change interventions' used to elicit a specific and more desirable response. ${ }^{14}$ In a separate study, Avery and colleagues ${ }^{15}$ also identified four specific BCTs from a recognized taxonomy, ${ }^{16}$ which were associated with increased physical activity: (1) prompt focus on (memory of) past success, (2) barrier identification/problem solving, (3) use of follow-up prompts, for example, motivational telephone calls, and (4) providing information on where and when to perform physical activity. This research suggests that GPs could optimize lifestyle counseling by applying these evidence-based strategies in their brief consultations. However, it remains unclear whether these or other BCTs are currently and commonly used in primary care consultations about physical activity with people with T2DM, and whether these are associated with achieving the recommended physical activity level in the 'real-world'.

Therefore, the aim of this study, in a general population of Australian adults with T2DM, was to examine associations between participants' self-reported engagement in the recommended level of physical activity and recall of specific physical activity-related interactions used by their GPs. ${ }^{7} 1718$

\section{RESEARCH DESIGN AND METHODS}

\section{Research design}

The Physical Activity Challenges and Triumphs (PACT) study was a cross-sectional, national, online survey (which participants completed at home) designed to assess psychosocial barriers and enablers of physical activity among Australian adults with T2DM. A paper version was available to participants on request.

The PACT study was overseen by a multidisciplinary advisory group which comprised experts in diabetes, behavioral sciences, exercise physiology, and primary care, as well as adults with T2DM.

The current study uses a selection of PACT data relating to recall of GP interactions about physical activity, selfreported physical activity, and relevant clinical and demographic variables (see the Measures section).

\section{Participants and sampling}

The PACT study inclusion criteria required participants to self-report a diagnosis of T2DM, be aged 18+ years, be resident in Australia, and be able to read and write in English without assistance. Eligibility for the current study required participants to have complete data on the measures of interest (described as follows).

Six hundred and sixty-six potential participants opened the online survey link, including $4(0.6 \%)$ who then declined to participate and $10(1.5 \%)$ who were excluded via the eligibility screening questions at the beginning of the survey. Participants with incomplete data were excluded: $77(11.6 \%)$ due to incomplete self-report physical activity data (ie, missing data on at least one subscale); 184 (27.6\%) due to exiting the survey before completing the GP interactions section; and $10(1.5 \%)$ due to incomplete data (about their diabetes $(n=7)$ or demographic characteristics $(n=3))$. The final sample for the current study was $n=381$.

\section{Procedure}

The study was advertised nationally through state-based diabetes member organization email lists and consumer magazines, staff and student email lists of two universities (one in Melbourne, one in Sydney), and diabetes industry email lists and social media pages.

The PACT survey was hosted by Qualtrics (a secure online survey platform) for a period of 3 months. All potential participants who visited the survey URL were presented with a plain language study description and online consent form. Those who provided informed consent (by clicking a box on the web page) were directed to an eligibility screening page, followed by the survey if eligible. Ineligible participants were screened out automatically and presented with a message thanking them for their interest and advising they were not eligible to take part. Participation was anonymous. At the conclusion of the study, participants were invited to enter their 
contact details on a separate website to enter a prize draw to win one of two iPads. The median survey completion time was $50 \mathrm{~min}$ (IQR: $38-76 \mathrm{~min}$ ). Two participants completed the questionnaire in hard copy rather than online.

\section{Measures}

The PACT survey comprised seven main sections, exploring: (1) current physical activity; (2) barriers, benefits and facilitators of physical activity; (3) motivation; (4) knowledge about physical activity; (5) sources of information and advice; (6) clinical characteristics; and (7) demographics. Each section included several questions measuring a broad range of constructs. Wherever possible, validated questionnaires were administered. In the absence of existing questionnaires, study-specific questions were developed for inclusion. Measures relevant to the current analysis are detailed as follows.

Physical activity: The key outcome of interest in this study was measured using the International Physical Activity Questionnaire-Short Form (IPAQ-SF). ${ }^{19}$ Respondents report the number of days that they have participated in vigorous and moderate physical activity and walked for at least $10 \mathrm{~min}$ at a time in the 7 days prior to completing the questionnaire as well as the amount of time spent on each activity in a usual day. Respondents also indicate the number of hours and minutes they usually spend sitting on a weekday in the past 7 days, although these data were not used in the current analysis. As per the recognized standard for the IPAQ-SF, responses are used to calculate physical activity MET-minutes (ie, metabolic equivalent, a measure of how much energy is expended during physical activity as a multiple of the resting metabolic rate). A MET-minute is computed by multiplying the relevant MET score (walking=3.3 METs, moderate physical activity $=4.0$ METs, vigorous physical activity $=8.0 \mathrm{METs}^{20}$ ) by the minutes performed for a given activity. Participants' total MET-minutes are calculated by summing across physical activity categories. For the current study, we transformed the total MET-minutes into a binary variable that represented meeting/exceeding the 600 MET$\mathrm{min} /$ week threshold or not meeting the threshold (ie, below 600 MET-min). Evidence indicates that this is the threshold for health benefits, equivalent to the national guidelines for physical activity (ie, approximately $150 \mathrm{~min}$ of moderate exercise per week). ${ }^{21}$

Interactions with GPs about physical activity: 14 studyspecific items asked participants if they recalled their GP ever (yes/no) using particular counseling techniques in relation to their physical activity. The purpose of the consultation, that is, diabetes related or not, was not specified. These items were informed by the findings of semistructured interviews with 28 adults $50 \%$ female; aged 28-77) with T2DM about the barriers and facilitators to being more physically active and the role of GPs in physical activity uptake. Fourteen techniques were identified that individuals with T2DM recalled their GP using or would like their GP to use. Twelve map onto Abraham and Michie's ${ }^{16}$ taxonomy of BCTs. A further two items (relating to instructing of a behavior), which are not part of the BCT taxonomy, were included due to their salience to the interview participants. The novel questionnaire was reviewed by the PACT advisory board prior to use.

Depressive symptoms: The WHO-5 Well-Being Index ${ }^{22}$ is a measure of general emotional well-being. Participants indicate how often each of the five positively worded statements has applied to them in the past 2 weeks on a 6 -point Likert scale ( $0=$ 'at no time' to $5=$ 'all of the time'). A total raw score is calculated by summing the item scores (range 0-25), with higher scores indicating better general emotional well-being. We used a score of $<13$ to indicate suboptimal well-being and depressive symptoms. ${ }^{23}$

Self-efficacy: The Barriers Self-Efficacy (BARSE) scale $\mathrm{e}^{17}$ consists of 13 items, each presenting a barrier to participation in physical activity. Participants indicate their confidence (from $0=$ "not at all confident' to $100=$ 'highly confident'), despite each of the presented barriers, that they could engage in the recommended level of physical activity over the next 3 months. The scale was modified, with the author's consent and in accordance with Australian Government Health Department's (2014) guidelines ${ }^{21}$ to replace 'exercise' with 'physical activity', and to use Australian Physical Activity and Sedentary Behaviour Guidelines of 30 min five times per week as opposed to three times per week. ${ }^{21}$ Total scores were calculated by summing the confidence ratings and dividing by the total number of items in the scale, resulting in a maximum possible self-efficacy score of 100 .

Self-reported demographic and clinical characteristics relevant to the current study include: age; gender; marital status; country of birth; education; employment status; household income; diabetes duration (years); insulin therapy use (insulin treated/non-insulin treated); most recent $\mathrm{HbAlc}(\%, \mathrm{mmol} / \mathrm{mol})$; height $(\mathrm{cm})$ and weight $(\mathrm{kg})$; and diagnosis of diabetes-related comorbidities (including: heart disease/heart attack, kidney damage, neuropathy, retinopathy, sexual dysfunction, stroke and vascular disease; summed as a total complication count from 0 to 7). Height and weight were used to calculate body mass index (BMI) to categorize participants into underweight, healthy, overweight and obese weight ranges. ${ }^{18}$

\section{Statistical analyses}

Statistical analysis was undertaken using IBM SPSS Statistics V.24. Descriptive statistics for all sample characteristics and variables of interest were calculated and reported.

Univariate tests were performed to examine differences in demographic, clinical or psychosocial characteristics by physical activity level $\left(\chi^{2}\right.$ tests for categorical variables; independent samples t-test or Mann-Whitney $\mathrm{U}$ test (for non-parametric data) for continuous variables).

Separate logistic regressions were conducted to explore the association between each of the 14 recalled GP interactions about physical activity (yes/no; independent variable) and level of physical activity $(<600$ and 
$\geq 600$ MET-min groups), adjusting for confounders of physical activity (as identified by the univariate analyses). Both unadjusted and adjusted parameter estimates are reported.

The assumptions of multicollinearity, goodness of fit and independence pertaining to the logistic regression models were tested and met. Tolerance statistics were assessed using the 1 /variance inflation factor with criteria for review set at a $<0.2$ threshold. The models were also tested for the influence and leverage of outliers using the Cooks distance statistic with criteria for assessment of influence set at values $>1$. No identified outliers returned a leverage value of significance.

Results are reported as mean \pm SD or n (\%). All statistical tests were two sided and differences were accepted as significant at $\mathrm{p}<0.05$.

\section{RESULTS}

\section{Characteristics of the sample}

Table 1 displays sociodemographic, psychosocial and selfreported clinical characteristics of the sample overall, and for both of the physical activity groups $(<600$ and $\geq 600$ MET-min). The mean age of participants was $62 \pm 10$ years; $55 \%$ were men, and $77 \%$ were born in Australia. Participants had been living with diabetes for $8 \pm 8$ years, most $(76 \%)$ were not using insulin, had been diagnosed with, on average, less than one diabetes-related complication $(81 \%)$, and approximately half $(51 \%)$ had a $\mathrm{BMI}$ in the obese range. Their HbAlc was $7.1 \% \pm 4.3 \%$ $(54 \pm 19 \mathrm{mmol} / \mathrm{mol})$ and $33 \%$ had suboptimal emotional well-being (WHO-5 <13), indicative of depressive symptoms. Of the 381 participants, 283 (74\%) reported meeting or exceeding $600 \mathrm{MET}$-min/week.

The associations between demographic, clinical or psychosocial variables and physical activity level $(<600$ MET-min vs $\geq 600$ MET-min) are shown in table 1 . Greater physical activity was associated with male gender $\left(\mathrm{X}^{2}(1)=4.01, \mathrm{p}=0.045\right)$, lower BMI $\left(\mathrm{X}^{2}(4)=22.0, \mathrm{p}<0.001\right)$, better emotional well-being (WHO-5; $\mathrm{X}^{2}(1)=25.56$, $\mathrm{p}<0.001$ ), greater self-efficacy (BARSE; $\mathrm{U}=6187.5$, $\mathrm{p}<0.001)$, and fewer comorbidities $(\mathrm{U}=11882.5, \mathrm{p}=0.02)$. As a result, these variables were considered confounders of physical activity level and were controlled for in the logistic regression analyses.

\section{Recalled GP interactions about physical activity}

Table 2 displays the proportion of participants who reported each type of GP interaction about physical activity for the total sample and split by physical activity level. Overall, the most common GP interaction related to information provision, with $73 \%$ of participants reporting that their GPs had given 'general advice about physical activity (eg, "physical activity is important")'. Interactions related to goal setting (14\%), self-monitoring of behavior $(16 \%)$ and relapse prevention (17\%) were least commonly reported.
Associations between GP interactions and physical activity level

Table 3 displays the unadjusted and adjusted parameter estimates of the logistic regression analyses. Univariate statistics reveal 5 of the 14 GP interactions were significantly associated with physical activity level over the last 7 days. After adjustment for confounders (gender, BMI, general emotional well-being and self-efficacy and diabetes-specific comorbidities), three statements relating to GPs' praise of their physical activity efforts significantly and independently associated with physical activity levels over the past 7 days. No other recalled GP interactions were significantly associated with participants' physical activity level.

Those who indicated they had been praised by their GP for making efforts to be active, for losing weight, or for lowering their blood glucose level through physical activity were between 1.75 and 2.1 times more likely to report achieving weekly MET-minutes $\geq 600$ in the preceding 7 days. A non-significant $(\mathrm{p}=0.07)$ positive trend was observed for the GP interaction 'Given you encouragement to be active', with those recalling this type of encouragement being 1.6 times more likely to report achieving weekly MET-minutes $\geq 600$ in the preceding 7 days.

\section{DISCUSSION}

Our findings demonstrate that Australian adults with T2DM who recall GP interactions about physical activity involving praise for their efforts to be physically active or associated clinical outcomes are more likely to self-report optimal physical activity level ( $\geq 600$ MET-min/week). Notably, other recalled GP interactions, including those most commonly reported by participants (general advice provision, encouragement to be active, and instruction to lose weight), were not significantly associated with self-reported optimal physical activity level. A trend was observed between GP interactions involving general encouragement and optimal physical activity level.

The praise-based GP interaction most strongly associated with optimal self-reported physical activity level was 'Praised your efforts to be active' which aligned with the BCT of 'provision of feedback on performance'. When praise was directed specifically at weight loss and reduced blood glucose level, which are the consequences of physical activity behavior, the association, while still significant, was weaker. This may indicate that praising the behavior, as opposed to the clinical outcome, is more beneficial. This is consistent with the findings of a review using a subsequent iteration of the BCT taxonomy. ${ }^{24}$ The review found that 'reinforcing effort or progress toward behaviour' was significantly associated with a higher level of physical activity in healthy adults, whereas techniques focusing on the consequences of a behavior (ie, an outcome) were not associated with physical activity level. As GP consultations typically focus on clinical outcomes rather than behaviors, ${ }^{9}$ this finding has implications for the way 
Table 1 Demographic, psychosocial and self-reported clinical characteristics for the total sample, and split by physical activity level (less than or at least $600 \mathrm{MET}-\mathrm{min} /$ week) $\dagger$

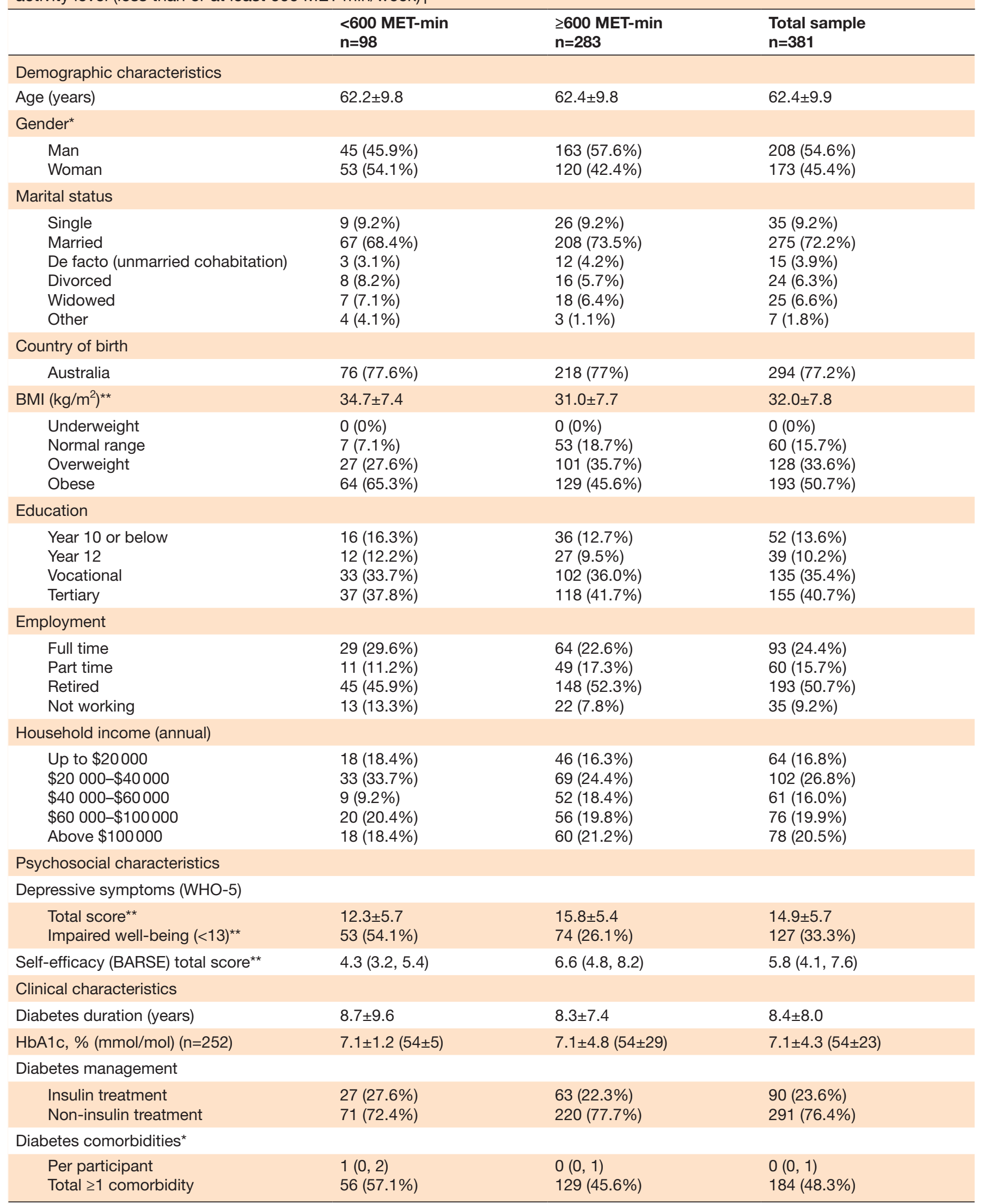

${ }^{*} \mathrm{p}<0.05 ;{ }^{* *} \mathrm{p}<0.01$

†All data are mean $\pm S D$, median (IQR) or $n(\%)$.

BARSE, Barriers Self-Efficacy; BMI, body mass index. 


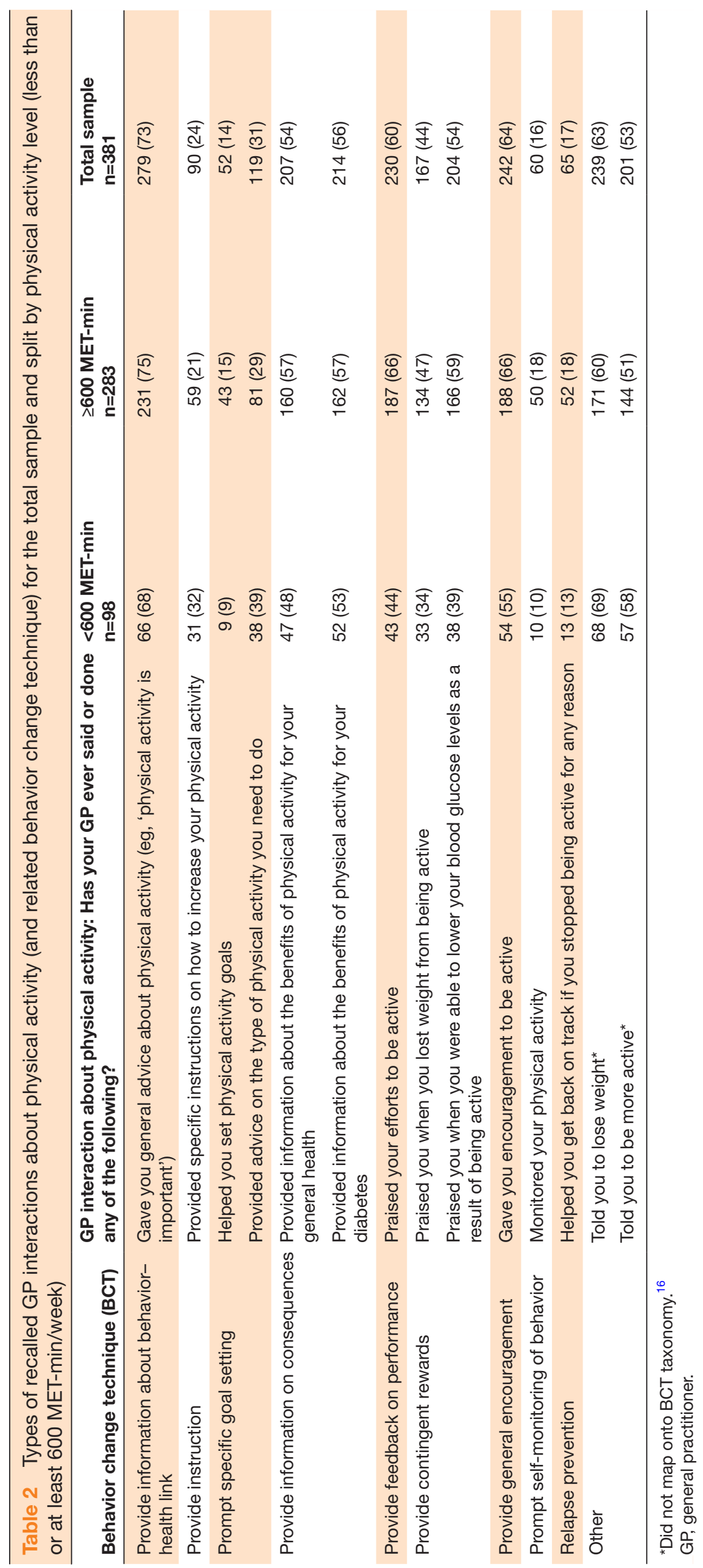




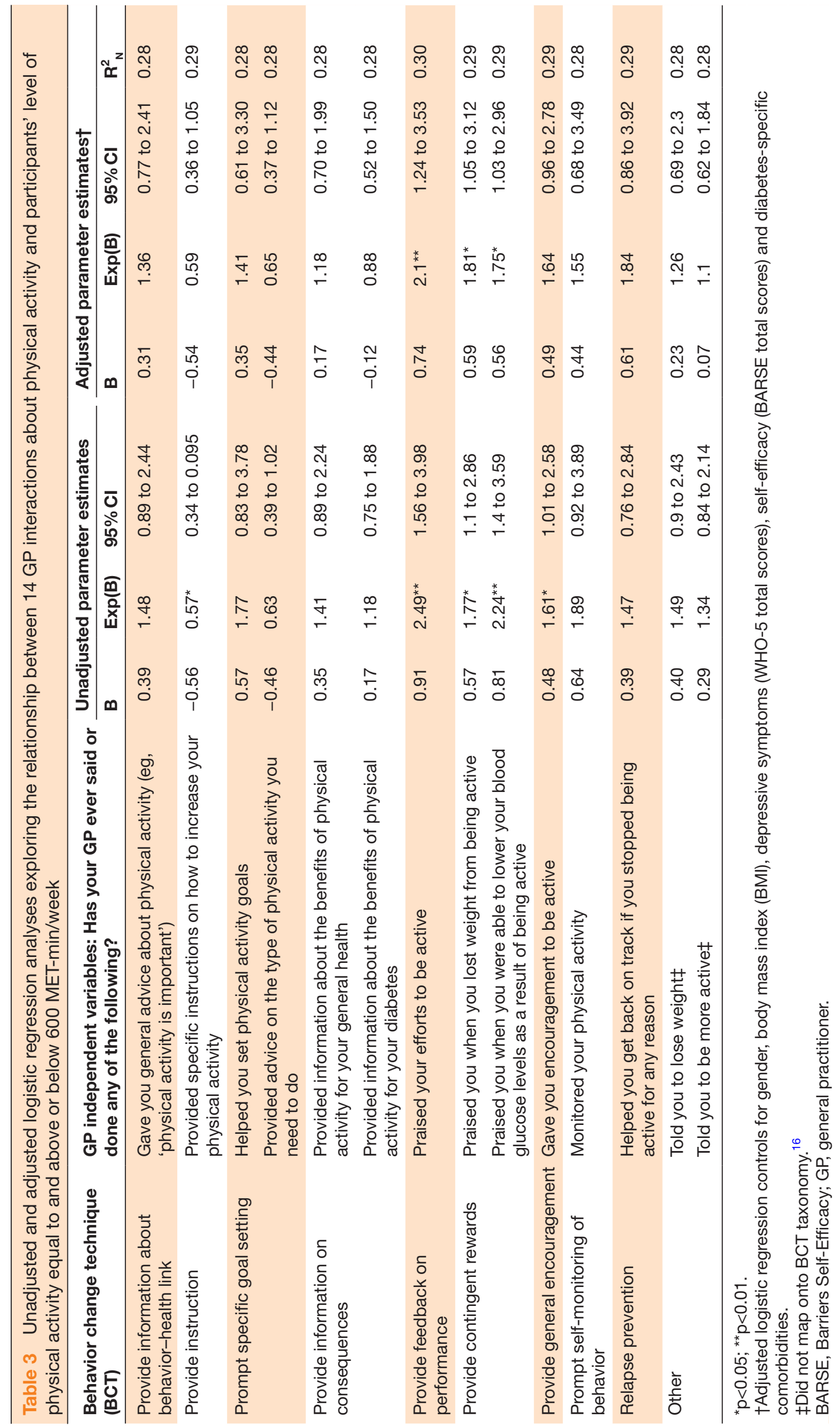


clinical diabetes care is delivered, and further research is warranted.

Prompting specific goal setting and self-monitoring of behavior has been shown previously to be associated with increased physical activity. ${ }^{25}{ }^{26}$ However, because these volitional BCTs pose a challenge to delivery due to their more time-intensive nature and dependence on participant enactment, GPs are more reliant on advice giving and direct persuasion. ${ }^{9}$ Indeed, these volitional BCTs were the least recalled interaction type in the current sample. This may be due to recall bias, but more likely suggests a training need for GPs to be able to prioritize, and deliver with high fidelity, the setting and reviewing of physical activity goals within primary care as a component of T2DM management for those needing to increase their physical activity. It is important to note that, in our study, 'praising efforts to be active' was effectively reinforcing effort towards the behavior, which might explain why this recalled technique was most strongly associated with optimal physical activity.

A review of 27 unique studies ${ }^{15}$ found that behavioral strategies associated with greater physical activity level in adults with type 2 diabetes related to prior success, problem solving, follow-up prompts and specific instructions regarding the physical activity to be performed. The heterogeneity of strategies and techniques associated with increased physical activity level suggests that GPs may need to employ a cluster of BCTs based on the needs of the individual, particularly with those not achieving the recommended guidelines for physical activity. Indeed, research has shown the synergistic impact of certain BCT clusters on increasing physical activity level (eg, action planning and coping planning) ${ }^{27}$ Furthermore, the way in which these are delivered (including the GP's consultation style) will influence the desired effect. In contrast, our analysis of techniques that individuals with T2DM recalled their GPs using has shown an association with positive feedback on performance and contingent rewards. However, the techniques employed in our study are not an exhaustive list and based only on the findings of interviews with people with T2DM.

The strengths of this study include the large sample of adults with T2DM who participated in the survey and its analysis focused on 'real world' physical activity level rather than intervention data. By investigating the techniques/strategies being used in primary care (as recalled by participants), and their associations with physical activity, this study offers much-needed insight into what is taking place within the primary care setting, over and above intervention-based research. ${ }^{15}$

However, several limitations of this study are acknowledged. Most notably, the cross-sectional nature of the data mean we are unable to comment on causation or the direction of the relationship between physical activity and the BCTs. Further, the recruitment of a selfselected convenience sample is likely to explain the fact that three-quarters of participants met or exceeded the national guidelines for physical activity. Such a high level is contrary to the proportion of the general population and T2DM population meeting recommended physical activity reported elsewhere, ${ }^{5}$ and may indicate that participants' motivation, capability or opportunity to be active was in contrast to the norm. Thus, these findings may not be representative of the associations between recalled GP interactions and physical activity among the wider population of adults with T2DM. However, given there was consistency between our study and the literature in the confounding variables that are typically associated with physical activity level across all participants (gender, ${ }^{28}$ BMI, ${ }^{29}$ depressive symptoms, ${ }^{30}$ self-efficacy, ${ }^{31}$ diabetes-specific comorbidities ${ }^{32}$ and other demographic variables), it follows that there may be consistency in the association of the identified BCTs with physical activity level.

We note that the BCTs assessed are not an exhaustive list nor reflective of the most up-to-date, and extended, BCT taxonomy published subsequent to the development of the PACT questionnaire. ${ }^{33}$ Precise mapping of the GP interactions onto the BCT taxonomy was not possible, as the GP interactions were generated from interviews with adults with T2DM. However, the latter is a strength, suggesting their face validity. We note that the data were reliant on participants' self-report, which may be subject to recall and social desirability biases. There is conflicting evidence regarding the reliability and validity of the IPAQ-SF for the measurement of self-reported physical activity level. ${ }^{20}{ }^{34}$ It may also be indicative of the level of missing physical activity data in this study and contributed to the high number of participants in the $\geq 600 \mathrm{MET}-\mathrm{min} /$ week category through overestimation of weekly physical activity level. Furthermore, participants' recall of GP interactions may be subject to conscious or unconscious recall bias, as demonstrated elsewhere. ${ }^{35} 36$ Finally, as the GPs were not the subject of this study, we do not know whether they were familiar with BCTs or have received any specific BCT training, or indeed whether participants' recall matches the GPs' intended strategies. Future research should incorporate objective measures of physical activity and observation (eg, video recording) of the GP consultation to minimize these potential biases.

Notwithstanding these limitations, particularly the cross-sectional data limiting causal inference, the current study suggests that providing positive feedback on performance and contingent rewards through praise might usefully form part of the lifestyle counseling provided by GPs during consultations with people with T2DM. Recently, recommendations for primary care intervention by Lamming $e t a l^{37}$ have indicated the need to develop and evaluate 'Very Brief Interventions' (VBI; 5 min or less) for more timely and realistic delivery in primary care settings, rather than longer interventions (of up to $30 \mathrm{~min}$ ), which were found to be prohibitive to deliver in primary care. Our findings indicate that further focus on the development and evaluation of primary care-based physical activity VBIs may be worthy of future exploration, as praising a person's efforts to be 
physically active is an approach that could be adopted in even the shortest of consultations. Given the association of BCTs 'feedback on performance' and 'providing contingent rewards' with physical activity level, further research is warranted to explore the use of this type of counseling using more robust measures and potentially applying them to other beneficial lifestyle modifications for effective T2DM management.

\section{CONCLUSIONS}

This study has demonstrated that, in the context of the realities of primary care, GPs' efforts and limited time could be well spent providing positive feedback about physical activity to adults with T2DM, particularly those not meeting physical activity recommendations. Specifically, GPs need to focus on performance and contingent rewards, such as praising the behavior, and the effort to be physically active in particular, rather than on the clinical outcomes achieved as a result of the behavior. While the success of primary care interventions for lifestyle modification relevant to T2DM management will be dependent on the needs of the individual, GPs can consider these simple techniques, as we have described, to promote positive behavioral changes among adults with T2DM, which will not negatively impact on the timeliness of consultations.

Acknowledgements We thank all the people who participated in the Physical Activity Challenges and Triumphs study.

Contributors RG conducted the data analyses and prepared the first draft of the manuscript. KM, JLB and JS conceived and conducted the study. JF was an advisory board member to the PACT study and contributed to the development of the interview protocol used to identify GP interactions that were subsequently included in the large-scale survey of adults with T2DM. JLB, EHT, JS, and KM provided input into the statistical analyses plan and interpretation of results. All authors reviewed, contributed to, and approved the manuscript.

Funding This work was supported by the Diabetes Australia Research Trust (DART; grant number Y13B-MOSK)

Competing interests None declared.

Patient consent for publication Not required

Ethics approval Ethics approval for the project was granted by the Human Research Ethics Committees of the Australian Catholic University (research project number: 2013 40N) and Deakin University (research project number: 2013 069).

Provenance and peer review Not commissioned; externally peer reviewed.

Data availability statement Data are available upon reasonable request.

Open access This is an open access article distributed in accordance with the Creative Commons Attribution Non Commercial (CC BY-NC 4.0) license, which permits others to distribute, remix, adapt, build upon this work non-commercially, and license their derivative works on different terms, provided the original work is properly cited, appropriate credit is given, any changes made indicated, and the use is non-commercial. See: http://creativecommons.org/licenses/by-nc/4.0/.

ORCID iD

Jane Speight http://orcid.org/0000-0002-1204-6896

\section{REFERENCES}

1 Conn VS, Hafdahl AR, Mehr DR, et al. Metabolic effects of interventions to increase exercise in adults with type 2 diabetes. Diabetologia 2007;50:913-21.
2 Gunton JE, Cheung NW, Davis TME, et al. A new blood glucose management algorithm for type 2 diabetes: a position statement of the Australian diabetes Society. Med J Aust 2014;201:650-3.

3 Franz MJ, Boucher JL, Rutten-Ramos S, et al. Lifestyle Weight-Loss Intervention Outcomes in Overweight and Obese Adults with Type 2 Diabetes: A Systematic Review and Meta-Analysis of Randomized Clinical Trials. J Acad Nutr Diet 2015;115:1447-63.

4 Pattyn N, Cornelissen VA, Eshghi SRT, et al. The effect of exercise on the cardiovascular risk factors constituting the metabolic syndrome: a meta-analysis of controlled trials. Sports Med 2013;43:121-33.

5 Lee I-M, Shiroma EJ, Lobelo F, et al. Effect of physical inactivity on major non-communicable diseases worldwide: an analysis of burden of disease and life expectancy. The Lancet 2012;380:219-29.

6 Colberg SR, Sigal RJ, Fernhall B, et al. Exercise and type 2 diabetes: the American College of sports medicine and the American diabetes association: joint position statement. Diabetes Care 2010;33:e147-67.

7 The Royal Australian College of General Practitioners. General practice management of type 2 diabetes: 2016-18. East Melbourne, VIc: RACGP 2016.

8 American Diabetes Association. Foundations of care and comprehensive medical evaluation. Sec. 32016.

9 Avery L, Charman SJ, Taylor L, et al. Systematic development of a theory-informed multifaceted behavioural intervention to increase physical activity of adults with type 2 diabetes in routine primary care: movement as medicine for type 2 diabetes. Implementation Science 2016;11:1-21.

10 Matthews A, Jones N, Thomas A, et al. An education programme influencing health professionals to recommend exercise to their type 2 diabetes patients - understanding the processes: a case study from Oxfordshire, UK. BMC Health Serv Res 2017;17:130.

11 Linetzky B, Jiang D, Funnell MM, et al. Exploring the role of the patient-physician relationship on insulin adherence and clinical outcomes in type 2 diabetes: insights from the mosaic study. $J$ Diabetes 2017:9:596-605.

12 Hébert ET, Caughy MO, Shuval K. Primary care providers' perceptions of physical activity counselling in a clinical setting: a systematic review. Br J Sports Med 2012;46:625-31.

13 Østbye T, Yarnall KSH, Krause KM, et al. Is there time for management of patients with chronic diseases in primary care? Ann Fam Med 2005;3:209-14.

14 Michie S, Johnston M. Theories and techniques of behaviour change: developing a cumulative science of behaviour change. Health Psychol Rev 2012;6:1-6.

15 Avery L, Flynn D, Dombrowski SU, et al. Successful behavioural strategies to increase physical activity and improve glucose control in adults with Type 2 diabetes. Diabetic Medicine 2015;32:1058-62.

16 Abraham C, Michie S. A taxonomy of behavior change techniques used in interventions. Health Psychol 2008;27:379-87.

17 McAuley $\mathrm{E}$. The role of efficacy cognitions in the prediction of exercise behavior in middle-aged adults. J Behav Med 1992;15:65-88

18 Heitmann BL. Evaluation of body fat estimated from body mass index, skinfolds and impedance. A comparative study. Eur J Clin Nutr 1990;44:831-7.

19 Craig CL, Marshall AL, Sjostrom M, et al. International physical activity questionnaire: 12 -Country reliability and validity. Medicine \& Science in Sports \& Exercise 2003;35:1381-95.

20 Silva-Batista C, Urso RP, Lima Silva AE, et al. Associations between fitness tests and the International physical activity questionnaireshort form in healthy men. J Strength Cond Res 2013;27:3481-7.

21 Brown WJ BA, Bull FC, Burton NW. Development of evidence-based physical activity recommendations for adults (18-64 years). Report prepared for the Australian government department of health. Canberra Australian Government Department of Health 2012.

22 Who collaborating centre for mental health. who (five) well-being index (1998 version). Frederiksborg General Hospital, DK-3400 Hillerød 1998.

23 Halliday JA, Hendrieckx C, Busija L, et al. Validation of the WHO-5 as a first-step screening instrument for depression in adults with diabetes: results from diabetes miles - Australia. Diabetes Res Clin Pract 2017:132:27-35.

24 Williams SL, French DP. What are the most effective intervention techniques for changing physical activity self-efficacy and physical activity behaviour--and are they the same? Health Educ Res 2011;26:308-22

25 Cradock KA, OLaighin G, Finucane FM, et al. Behaviour change techniques targeting both diet and physical activity in type 2 diabetes: a systematic review and meta-analysis. Int J Behav Nutr Phys Act 2017;14. 
26 Hankonen N, Sutton S, Prevost AT, et al. Which behavior change techniques are associated with changes in physical activity, diet and body mass index in people with recently diagnosed diabetes? Ann Behav Med 2015;49:7-17.

27 Sniehotta FF, Schwarzer R, Scholz U, et al. Action planning and coping planning for long-term lifestyle change: theory and assessment. Eur J Soc Psychol 2005;35:565-76.

28 Bauman A, Bull F, Chey T, et al. The International prevalence study on physical activity: results from 20 countries. Int J Behav Nutr Phys Act 2009;6.

29 Cameron AJ, Welborn TA, Zimmet PZ, et al. Overweight and obesity in Australia: the 1999-2000 Australian diabetes, obesity and lifestyle study (AusDiab). Med J Aust 2003;178:427-32.

30 Win S, Parakh K, Eze-Nliam CM, et al. Depressive symptoms, physical inactivity and risk of cardiovascular mortality in older adults: the cardiovascular health study. Heart 2011;97:500-5.

31 Stutts WC. Physical Activity Determinants in Adults:Perceived Benefits, Barriers, and Self Efficacy. Am Ass Occupational Health Nurses J 2002;50:499-507.
32 Gregg EW, Gerzoff RB, Caspersen CJ, et al. Relationship of walking to mortality among US adults with diabetes. Arch Intern Med 2003;163:1440-7.

33 Michie S, Richardson M, Johnston M, et al. The behavior change technique taxonomy (V1) of 93 hierarchically clustered techniques: building an international consensus for the reporting of behavior change interventions. Ann Behav Med 2013;46:81-95.

34 Lee PH, Macfarlane DJ, Lam TH, et al. Validity of the International physical activity questionnaire short form (IPAQ-SF): a systematic review. Int J Behav Nutr Phys Act 2011;8.

35 Parkin T, Skinner TC. Discrepancies between patient and professionals recall and perception of an outpatient consultation. Diabetic Medicine 2003;20:909-14.

36 Skinner TC, Barnard K, Cradock S, et al. Patient and professional accuracy of recalled treatment decisions in out-patient consultations. Diabetic Medicine 2007;24:557-60.

37 Lamming L, Pears S, Mason D, et al. What do we know about brief interventions for physical activity that could be delivered in primary care consultations? A systematic review of reviews. Prev Med 2017;99:152-63. 